\title{
The impact of COVID-19 on patients of Italian mental health supported accommodation services
}

\author{
Alessandra Martinelli ${ }^{1,2}$ (D) Mirella Ruggeri ${ }^{1,2}$ (C) \\ Received: 5 May 2020 / Accepted: 9 June 2020 / Published online: 25 July 2020 \\ c) Springer-Verlag GmbH Germany, part of Springer Nature 2020
}

At this pandemic time, each person has been experiencing a feeling of being at risk of a sudden and invincible attack of an invisible enemy. This is a sensation that in psychopathology might be called a 'predelusional state' [1]. With daily observation, in some mental health supported accommodation services in the area of Verona, we have found that users with severe mental illness (SMI) [2] have reacted differently to the pandemic lockdown, depending on the specific mental health disorder they suffer.

Overall, severe psychotic patients with depersonalization symptoms are those who are showing more difficulties in following basic rules to avoid infection, such as keeping selfhygiene, security distances, and masks. Particularly, people with a diagnosis of schizophrenia, during the first two weeks of lockdown, were relaxed and quiet, probably because of the under-stimuli situation characterized by a suspension of individual and group activities both inside and outside the supported accommodation, supporting the typical research of social withdrawal of these patients.

However, from the third week of lockdown, patients with schizophrenia have started to lose control mostly on positive symptoms, showing disorientation and an increased difficulty to maintain a connection with reality. Generally, the typical delusional topic of each patient has strongly emerged. Specifically, schizoaffective patients have shown peaks of manic symptoms with sexual disinhibition, while psychotic patients characterized by negative symptoms have developed loneliness feelings, with increasing protective psychotic regressions: initially, they need support to take

Mirella Ruggeri

mirella.ruggeri@univr.it

Alessandra Martinelli

alessandra.martinelli@univr.it

1 Section of Psychiatry, Department of Neuroscience, Biomedicine and Movement, University of Verona, Verona, Italy

2 Verona Hospital Trust, AOUI, Verona, Italy care of self-hygiene; then they need support to carry on basic daily life activities such as eating and drinking. Other psychotic patients, after 2 weeks of lockdown, have started to reschedule their daily plan with compulsive activities, such as excessive physical activities or eating, while others have increased specific unusual thoughts focussed on the fear of being infected by others or to infect others.

On the other hand, patients suffering from anxiety and depression symptoms have reacted with a worsening of their state, needing more caregiving and sometimes with dysphoria because of the frustration of the impossibility of going out.

Patients with a diagnosis of borderline personality disorder have shown a range of different reactions: from locking themselves in their rooms hypothesizing to have had close contacts with infected people-interpretable as an unconscious manner to protect themselves from the incapability of respecting rules-to put themselves in risky situations, showing a propensity to 'dare' the virus.

Patients with a diagnosis of psychotic personalities, most characterized by social withdrawal and self-social exclusion, have shown a stable good condition for the whole lockdown duration as if they were relieved of a low requirement to perform social activities.

Service users with obsessive-compulsive disorders offered to be of help in keeping hygiene even of common spaces of supported accommodation.

Patients with a continuous manic state were those with more difficulties in keeping the rules of the lockdown, showing issues in understanding the real reason for the necessary lockdown.

However, after more than 1 month of the pandemic lockdown, despite described users' psychopathological difficulties and the obligation to stay every day together, users have shown growing reciprocal solidarity, taking care of each other in daily obstacles and reducing the sometimes usually high level of quarrel or aggressivity. 
Furthermore, the staff have become aware of the specificities in the different reactions by users with different psychopathological characteristics and have learned how to modulate the reactions of patients, to reduce the behavioral components at greater risk of crisis, and, at the same time, to employ guests in the domestic activities that were the most appropriate to their mental state.

In conclusion, this pandemic time has given the opportunity to better understand the limitations, needs and also the resources of people with SMI in mental health supported accommodation services. Against any assumption, our users have shown a higher resilience than expected, and tender solidarity among each other, trying to overcome this difficult period together.

Furthermore, the overall impact of the pandemic state on the psychopathology of our users has shown the adequacy of the fairly consolidated theoretical-practical corpus of psychiatric rehabilitation developed in the Italian mental health community care since deinstitutionalization, and, above all, in the last 10 years. For example, the reason for psychopathological crises in people with severe psychosis might be found in a vacuum in their lives caused by the lack of daily scheduled activities, a milestone of rehabilitation therapeutic programs, confirming that restraint and psychopharmacological therapies cannot respond to personal and social needs of people with SMI [3, 4].

Finally, this experience carried out in a protected supported accommodation services and the strategies implemented by the mental health staff might be a model for dealing with behavioral aspects in the general population with beginning dysfunctional reactions to stress, uncertainty, and limitations of the pandemic. It will certainly be of fundamental importance shortly not only to monitor the possible aggravations of those who already suffer from mental health disorders, but also to carefully identify the specificity of the new cases and their implications in terms of functioning and to activate early monitoring of the distress phenomena in the general population with a preventive approach.

\section{Compliance with ethical standards}

Conflict of interest The authors declare that they have no competing interests.

\section{References}

1. Callieri B (1964) Psicopatologia classica e psichiatria interpersonale di fronte alla perplessità schizofrenica. Psichiatr Gen dell'Età Evol 2:1

2. Martinelli A, Iozzino L, Ruggeri M et al (2019) Mental health supported accommodation services in England and in Italy: a comparison. Soc Psychiatry Psychiatr Epidemiol 54(11):1419-1427

3. Liberman RP (2008) Recovery from disability: manual of psychiatric rehabilitation. American Psychiatric Publishing Inc., Washington, DC

4. Killaspy H, Harden C, Holloway F et al (2005) What do mental health rehabilitation services do and what are they for? A national survey in England. J Ment Heal 14(2):157-165 